\title{
ESTRUCTURA POR EDADES Y REPRODUCCION DE TRES POBLACIONES DE SARGASSUM SINICOLA (PHAEOPHYTA, FUCALES) EN LA BAHIA DE LA PAZ, GOLFO DE CALIFORNIA
}

\author{
JULIO ESPINOZA \\ Centro de Investigaciones Biológicas de Baja California Sur, A.C. \\ Apartado Postal No. 128 \\ 23060 La Paz, Baja California Sur, México
}

\begin{abstract}
RESUMEN
La edad máxima de las plantas de Sargassum sinicola en la Bahía de La Paz, Golfo de California, México, es de tres años. Esta edad se estimó midiendo la longitud del eje central.

La estructura por edades de Sargassum sinicola en profundidades someras (1-2 $\mathrm{m}$ ) de El Cajete y Las Pacas y en un sitio "Profundo" (11 m) fue dominada por las plantas de un año de edad, o más jóvenes. Esta clase incluyó $96.9 \%, 91.6 \%$ y $96.4 \%$ del total de individuos muestreados, respectivamente. Se discute la estrategia reproductiva que $S$. sinicola posee para poder mantenerse como dominante en el área de estudio, ya que cada año tiene que reemplazar la mayor parte de sus individuos.

Se encontró que la reproducción de Sargassum sinicola estuvo relacionada más bien con la talla y no con la edad de los individuos.
\end{abstract}

\begin{abstract}
The maximum age reached by plants of Sargassum sinicola in La Paz Bay, Gulf of California, Mexi$\mathrm{Co}$, is of three years. Age was estimated by measuring the length of the main axis.

Age structure of Sargassum sinicola at 1-2 m depth in El Cajete and Las Pacas, and in a "Profundo" $(11 \mathrm{~m})$ site, was dominated by plants one year old, or youngers. This class comprised $96.9 \%, 91.6 \%$ and $96.4 \%$ of the total population sampled, respectively. The reproductive strategy that $S$. sinicola must exhibit in order to maintain a dominant role in the community is discussed. $S$. sinicola must replace the great majority of individuals in a population every year. It was found that reproduction in Sargassum sinicola was more related to plant size than to individual age.
\end{abstract}

\section{INTRODUCCION}

El patrón fenológico de la macroalga marina Sargassum sinicola Setchell et Gardner comprende una serie de etapas, incluyendo la época de crecimiento máximo, el período de reproducción, y la muerte de ramificaciones que crecen al tamaño máximo. Las partes perennes de $S$. sinicola incluyen un rizoide, un eje central o estipe y las ramificaciones que no mueren al alcanzar la planta su talla máxima. Las ramificaciones que van muriendo dejan una cicatriz en el eje central, en forma de protuberancia. En base a esta observación, Espinoza Avalos y Rodríguez Garza (1985) encontraron que el número de cicatrices dejadas en el eje central permitía determinar la edad de esta macroalga café. Este método ya había sido utilizado con anterioridad por Umezaki (1983). Espinoza Avalos y Rodríguez Garza (1985) también encontraron que la distancia del eje central, medida desde su unión con el 
rizoide hasta encontrar la primera ramificación primaria (de acuerdo a Jensen, 1974), representaba otro método para determinar la edad de $S$. sinicola. Las menciones posteriores que se hagan respecto al eje central se referirán a esta última medida.

Por otra parte, Espinoza y Rodríguez $(1987 ; 1989)$ encontraron que una población de Sargassum sinicola crecía más lentamente, alcanzaba menor tamaño, se reproducla menos y su morfología era diferente con respecto a otra que se encontraba a la misma profundidad, pero a $7 \mathrm{~km}$ de distancia. Los anteriores autores propusieron que la luz era el principal factor que originaba esas diferencias.

Los objetivos de este estudio fueron: 1) determinar el tiempo de vida de esta especie, 2) conocer si las dos poblaciones ya mencionadas presentan diferencias en su composición por edades, lo cual podría ayudar a esclarecer sus diferencias fenológicas, 3) estudiar la composición por edades de $S$. sinicola encontrada a $11 \mathrm{~m}$ de profundidad para compararla con las dos primeras poblaciones, que crecen en sitios someros, y 4) conocer si su madurez reproductiva se relaciona con la edad o con el tamaño de las plantas.

\section{AREA DE ESTUDIO}

Las tres zonas de estudio se localizan en la parte suroeste de la Bahía de La Paz, Golfo de California, México (Fig. 1). El Cajete está ubicado a aproximadamente $7 \mathrm{~km}$ al norte de Las Pacas. La profundidad de colecta en estas zonas fue de 1-2 m. El tercer sitio se encuentra a aproximadamente $1 \mathrm{~km}$ de la costa, a una profundidad de $11 \mathrm{~m}$, y se le denominará "Profundo" de aquí en adelante. Las tres zonas están colindadas por arena. La población de S. sinicola en El Cajete ocupa una banda litoral de aproximadamente 15-20 m de ancho y $150 \mathrm{~m}$ de largo. El manto de algas en Las Pacas ocupa una superficie similar, aunque irregular en sus contornos. La superficie ocupada por $S$. sinicola en el sitio "Profundo" se desconoce. En este último lugar las plantas se encuentran adheridas a rocas aisladas, no mayores de $30 \mathrm{~cm}$ de diámetro.

\section{METODOLOGIA}

Determinación del tiempo de vida

Durante cada muestreo se colectaron selectivamente plantas de Sargassum sinicola que presentaban los ejes centrales más largos ( $y$ por ello los individuos de mayor edad), utilizando equipo de buceo autónomo. En el mes de mayo de los años 1985, 1986 y 1987 se colectaron 61, 58 y 66 plantas, respectivamente, del sitio denominado Las Pacas. Una población completa de $S$. sinicola de Las Pacas se habla reinstalado en 1984, posterior a un disturbio catastrófico ocurrido en esa zona (Espinoza Avalos y Rodríguez Garza, 1985), por lo que la mayor edad posible de las plantas en mayo de 1985 era de un año. De igual manera, la edad máxima posible de estas plantas para mayo de 1986 y 1987 era de 2 y 3 años, respectivamente.

Las plantas se separaron del fondo rocoso con una espátula y posteriormente fueron llevadas al laboratorio donde se les contó el número de cicatrices en el eje central, y el largo del mismo. 


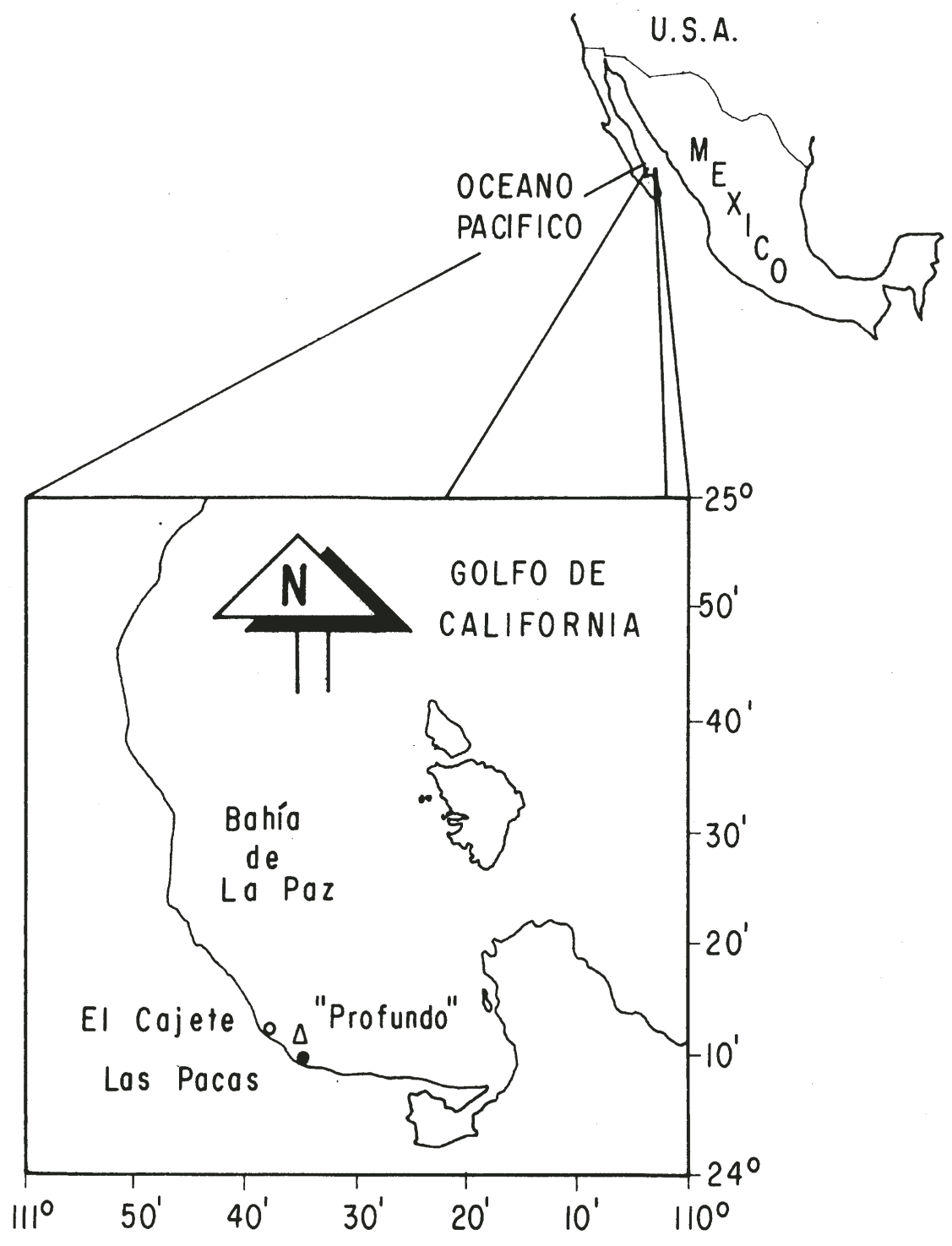

Fig. 1. Localización de El Cajete (0), Las Pacas ( $)$ y el sitio "Profundo" ( $\Delta)$, en la Bahía de La Paz, Golfo de California, México. 
Estructura por edades, tallas y reproducción

En mayo de 1987 se colectaron por medio de buceo autónomo todas las plantas de Sargassum sinicola encontradas en 10 cuadrados de $20 \mathrm{~cm}$ de lado, de la población de Las Pacas. Muestreos preliminares indicaron que el número de plantas colectadas no era suficiente al utilizar cuadrados de 10 y $15 \mathrm{~cm}$ de lado. El tamaño de muestra utilizado en El Cajete y en el sitio "Profundo" fue igual que en Las Pacas. Se midió la longitud del eje central a todas las plantas colectadas en los 10 cuadrados de cada sitio. La talla total, así como su estado reproductivo (determinado en función de la presencia o ausencia de receptáculos), se obtuvo para las plantas encontradas en 5 cuadrados de los dos primeros sitios, y en 6 para el sitio "Profundo".

\section{RESULTADOS}

La longitud del eje central presentó una correlación positiva $\left(r^{2}=0.85, n=185\right)$ con el número de cicatrices encontradas en el mismo eje al graficar los valores para 1985, 1986 y 1987 (Fig. 2). La edad de las plantas de Sargassum sinicola se fijó empíricamente en las clases I, II y III (Fig. 2), correspondientes a 1, 2 y 3 años de edad, respectivamente. Para ello se tomó en consideración el punto medio del traslape de datos entre cada clase de edad. Así, la longitud que se utilizó para delimitar la edad en 1, 2 y 3 años correspondió a plantas menores de $2.1 \mathrm{~cm}$, entre 2.2 y $3.6 \mathrm{~cm}$, y mayores de $3.6 \mathrm{~cm}$, respectivamente; y el número de cicatrices en el eje central fue de 1-13, 14-21 y mayor de 21 para las edades de 1,2 y 3 años, respectivamente. La primera clase de edad contenía individuos con un año de edad o más jóvenes, ya que en los meses anteriores al muestreo hubo reclutamiento. Los valores máximos encontrados fueron de $6.9 \mathrm{~cm}$ de longitud y de 45 cicatrices.

La clase de edad I comprendió $96.9 \%, 91.6 \%$ y $96.4 \%$ del total de plantas de Sargassum sinicola en El Cajete, Las Pacas y el sitio "Profundo", respectivamente (Fig. 3A). Las plantas de 2 años de edad representaron un bajo porcentaje en los tres sitios y la participación de la clase III fue menor de $2 \%$ en El Cajete y Las Pacas. En el sitio "Profundo" no se encontraron plantas de 3 años de edad.

Ninguna de las pocas plantas de 3 años encontradas en El Cajete y Las Pacas se estaba reproduciendo en mayo de 1987 (Fig. 3). Tampoco se encontraron individuos $(n=7)$ de 2 años de El Cajete en reproducción. Sin embargo, en la clase I siempre hubo plantas fértiles en los tres sitios de muestreo (Fig. 3B). Estos datos no presentaron ningún patrón definido entre fertilidad y edad (Fig. 3).

Para buscar si existla relación entre la talla y la madurez reproductiva en Sargassum sinicola, primeramente se definieron cuatro clases de talla $(1,<15 \mathrm{~cm} ; 2,15.1-30 \mathrm{~cm} ; 3$, $30.1-45 \mathrm{~cm} ; 4>45 \mathrm{~cm}$ ). Se observó un patrón general, con mayor porcentaje de individuos menores de $15 \mathrm{~cm}$, y menor presencia de plantas mayores de $45 \mathrm{~cm}$ (Fig. 4A). En El Cajete y el sitio "Profundo" ninguna planta de la clase I se encontraba en reproducción y sólo un ínfimo porcentaje de los individuos de ese tamaño lo estaba en Las Pacas. Pcr otra parte, en los tres sitios de muestreo se encontró que el porcentaje de individuos fértiles de $S$. sinicola fue mayor conforme la talla se incrementó (Fig. 4B).

El mayor porcentaje de individuos reproductivos se encontró entre aquellos de mayor tamaño (Fig. 4). 


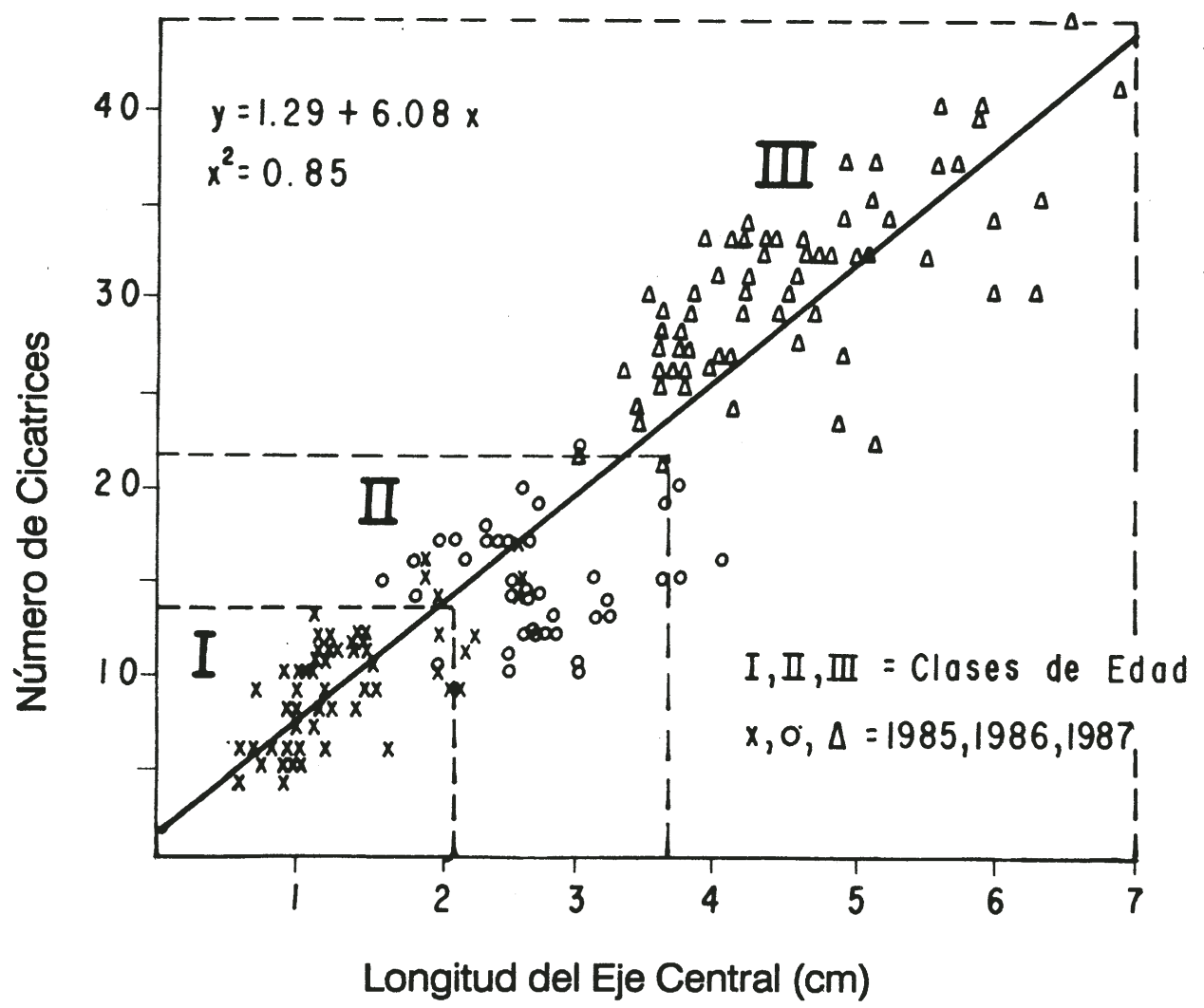

Fig. 2. Relación entre la longitud del eje central $(\mathrm{cm})$ y el número de cicatrices del eje central para plantas de Sargassum sinicola colectadas en 1985 (clase de edad I=1 año de edad), 1986 (clase de edad II=2 años de edad) y 1987 (clase de edad III=3 años de edad) en Las Pacas, Bahía de La Paz, Golfo de California. 


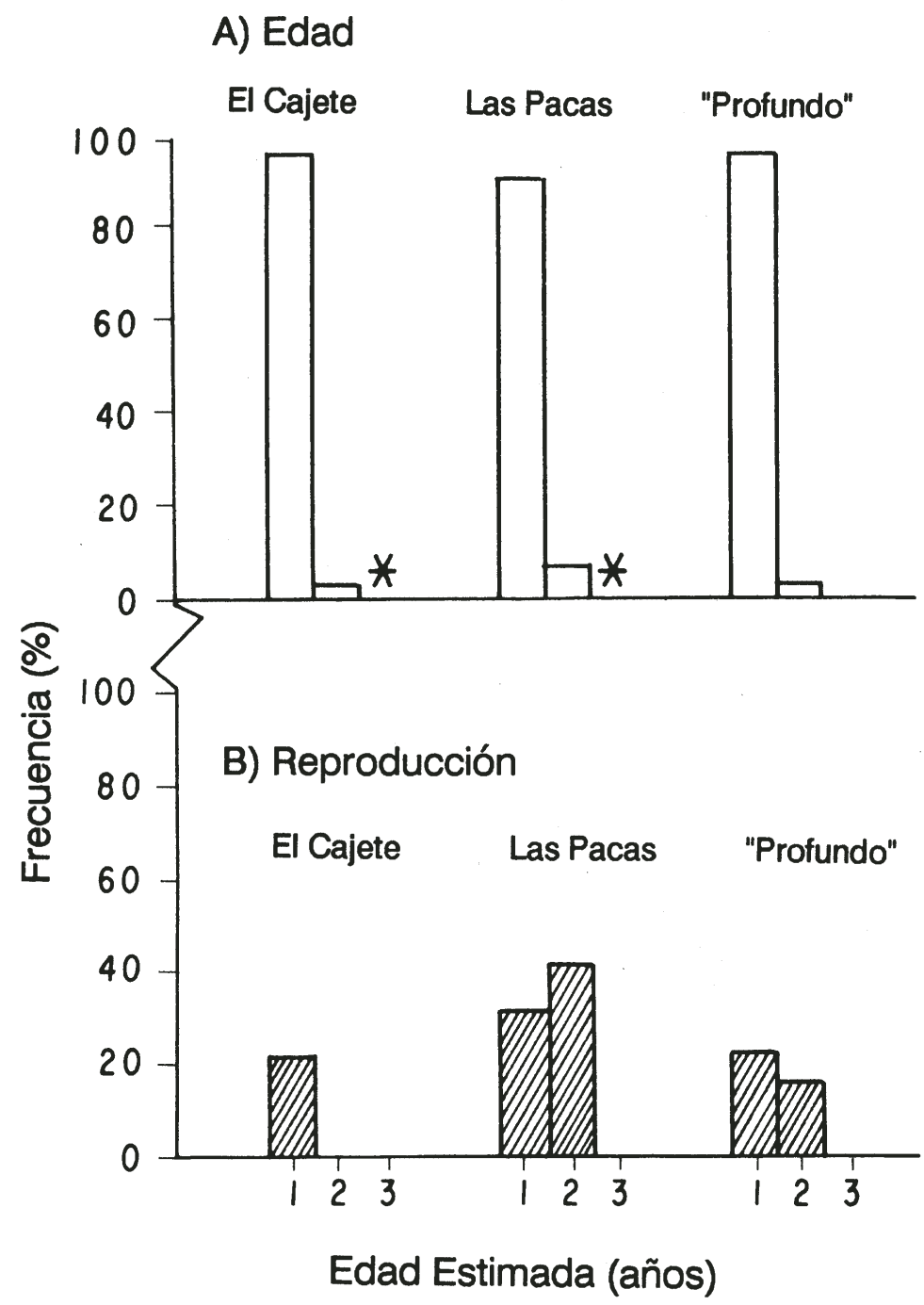

Fig. 3. Frecuencia de clases de edad (A) y de reproducción (B) de Sargassum sinicola en El Cajete $(n=612)$, Las Pacas ( $n=725$ ) y sitio "Profundo" ( $n=393$ ), en la Bahía de La Paz, Golfo de California. El tamaño de muestra indicado entre paréntesis para cada sitio corresponde para la gráfica de edad (3A). El tamaño de muestra para la gráfica de reproducción (3B) fue de 218,352 y 238 , respectivamente (ver detalles en metodología). " Representa un valor de porcentaje menor de $2 \%$. 


\section{DISCUSION}

Los resultados de este estudio confirman que la edad de Sargassum sinicola puede calcularse por la longitud del eje central o por el número de cicatrices, como lo sugirieron Espinoza Avalos y Rodríguez Garza (1985). Sin embargo, las determinaciones de edad de $S$. sinicola utilizando la longitud del eje central permiten llevar a cabo las mediciones fácilmente y, si es necesario, sin destruir ni desprender la planta del fondo rocoso.

Los valores máximos de longitud del eje central y de número de cicatrices encontrados en este estudio para las plantas correspondientes a la clase de edad III $(6.9 \mathrm{~cm}$ y 45 cicatrices) fueron similares a los valores máximos $(6.3 \mathrm{~cm}$ y 44 cicatrices) encontrados por Espinoza Avalos y Rodríguez Garza (1985) en un muestreo llevado a cabo para una población de edades desconocidas. Por ello se concluyó que el tiempo de vida máximo de Sargassum sinicola en la Bahía de La Paz, Golfo de California, es de 3 años. Esta longevidad es relativamente baja, comparada con otros valores encontrados en la literatura: 4 años para S. muticum (Critchley, 1981) y para $S$. hemiphyllum (Umezaki, 1984) y 7 años para $S$. confusum (Nabata et al., 1981). No se encontraron plantas de $S$. sinicola de tres años de edad en el sitio "Profundo", pero sí las hubo en El Cajete y Las Pacas (Fig. 3A). Sin embargo, esta diferencia no se puede considerar significativa debido al escaso número de plantas de 3 años encontradas en los dos últimos sitios (Fig. 3A).

La estructura por edades de Sargassum sinicola fue dominada por las plantas de la clase I, presentándose un patrón muy similar en los tres sitios de muestreo (Fig. 3A). Por esta razón, no es posible relacionar los datos de edad con diferencias fenológicas encontradas anteriormente entre las plantas de El Cajete y Las Pacas (ver Espinoza y Rodríguez, $1987 ; 1989)$. Es importante señalar, sin embargo, que $S$. sinicola mantuvo una estructura de edades similar en condiciones ambientales relativamente diferentes.

La clase de edad más frecuente encontrada en otras especies de Sargassum ha sido mayor, en comparación con la determinada en $S$. sinicola: 1 y 2 años en $S$. muticum (Critchley, 1981) y en S. ringgoldianum (Yoshida, 1960); y 2 y 3 años en $S$. miyabe (Umezaki, 1983). Es posible que la clase de edad más frecuente en estas especies sea superior a la de $S$. sinicola debido a que su longevidad también es mayor, como ya se menciono.

La dominancia de plantas de S. sinicola en la clase de edad I sugiere una estrategia reproductiva eficiente, que le permite mantener una dominancia ecológica, en términos de biomasa (como ocurre en la Bahía de La Paz), a pesar de que cada año tiene que reemplazar a la mayoría de los individuos de la población. Esta observación ya fue mencionada por Espinoza y Rodríguez (1987), quienes indicaron que $S$. sinicola puede reproducirse ya sea sexualmente, o vegetativamente por medio del rizoide. En las plantas de El Cajete, por otro lado, es factible la presencia de dos picos de reproducción en un año. Es decir, una vez que $S$. sinicola se establece, puede aprovechar sus alternativas reproductivas para ser una fuerte competidora por espacio.

Aunque la estructura por edades de Sargassum sinicola no presentó una relación bien definida con el número de plantas fértiles (Fig. 3), esta relación sí se presentó claramente con respecto a la talla (a mayor talla alcanzada, mayor porcentaje de plantas reproductivas, Fig. 4). Lo anterior representa otro ejemplo del hecho que la fertilidad está más relacionada con el tamaño del individuo que con la edad, tal como ocurre en Laminaria longicruris (Chapman, 1986). 


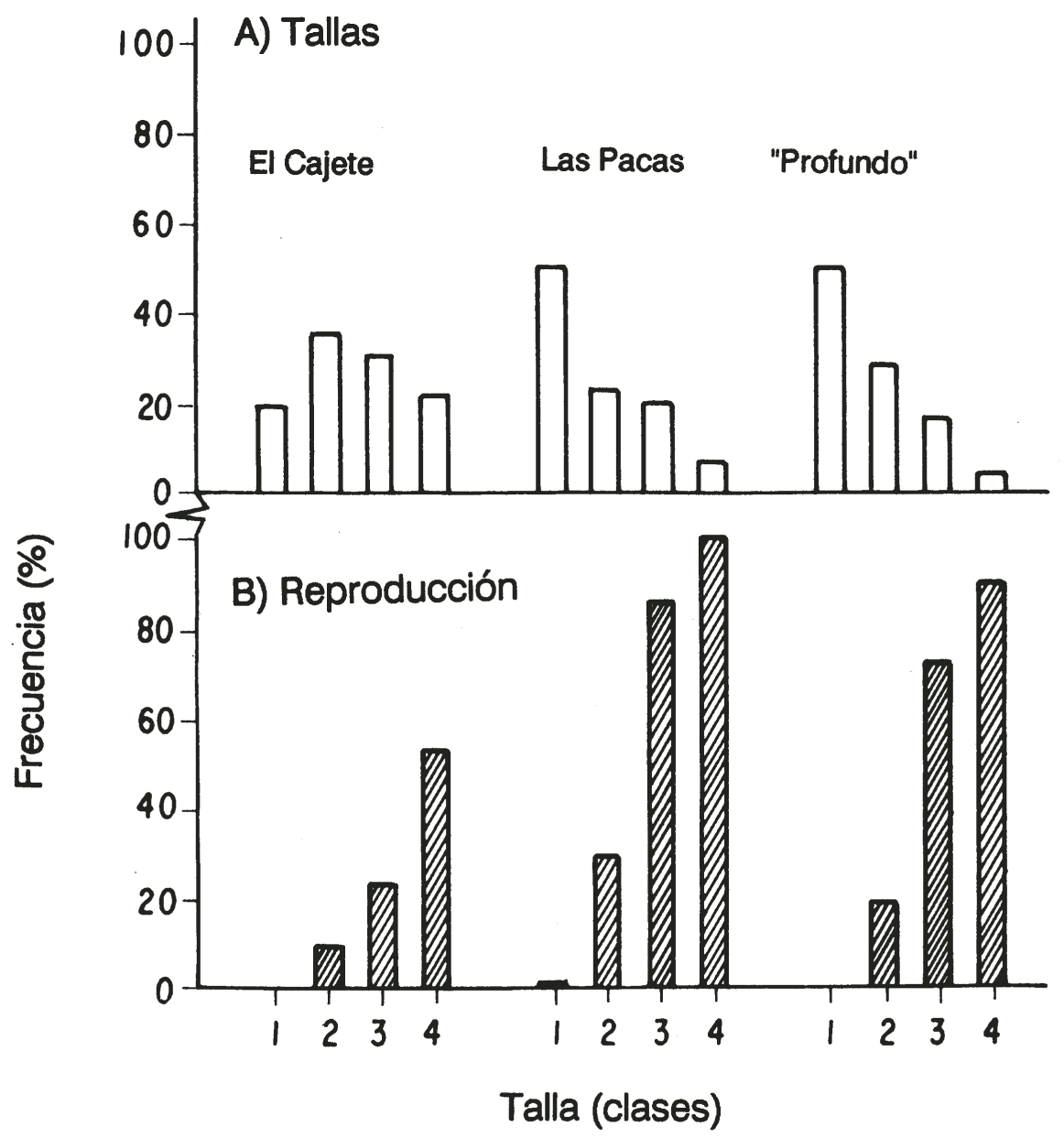

Fig. 4. Frecuencia de clases de tallas $(A: 1,<15 \mathrm{~cm} ; 2,15.1-30 \mathrm{~cm} ; 3,30.1-45 \mathrm{~cm} ; 4,>45 \mathrm{~cm})$ y de reproducción (B) en Sargassum sinicola de El Cajete $(n=218)$, Las Pacas $(n=352)$ y sitio "Profundo" $(n=238)$, en la Bahla de La Paz, Golfo de California. 
Por otra parte, se pudo observar que la edad no presentó ninguna correlación con la talla de $S$. sinicola en El Cajete $\left(r^{2}=0.04, n=218\right)$, en Las Pacas $\left(r^{2}=0.20, n=352\right)$, $n$ i en el sitio "Profundo" $\left(r^{2}=0.18, n=238\right)$. Esto ocurre porque cada vez que se presenta la época optima de crecimiento, la mayoría de los individuos de $S$. sinicola pueden crecer al máximo, independientemente de su edad.

\section{AGRADECIMIENTOS}

El autor agradece el apoyo logístico dado por el Dr. D. Lluch Belda. En especial agradece la ayuda brindada por el B.M. H. Rodríguez Garza. El contenido del manuscrito mejoró substancialmente con las sugerencias aportadas por dos revisores anónimos.

\section{LITERATURA CITADA}

Chapman, A.R.O. 1986. Age versus stage: an analysis of age and size-specific mortality and reproduction in a population of Laminaria longicruris Pyl. J. Exp. Mar. Biol. Ecol. 97:113-122.

Critchley, A. 1981. Age determination of Sargassum muticum (Yendo) Fensholt. Brit. Phycol. J. 16:134.

Espinoza-Avalos, J. \& H. Rodríguez-Garza. 1985. Observaciones preliminares de Sargassum sinicola Setchell et Gardner (Phaeophyta) en la Bahía de La Paz, Golfo de California. Ciencias Marinas 11:115-120.

Espinoza, J. \& H. Rodríguez. 1987. Seasonal phenology and reciprocal transplantation of Sargassum sinicola Setchell et Gardner in the southern Gulf of California. J. Exp. Mar. Biol. Ecol. 110:183-195.

Espinoza, J. \& H. Rodríguez. 1989. Crecimiento de Sargassum sinicola Setchell et Gardner (Phaeophyta) en la parte sur del Golfo de California, México. Ciencias Marinas 15:141-149.

Jensen, J.B. 1974. Morphological studies in Cystoseiraceae and Sargassaceae (Phaeophyceae) with special reference to apical organization. Univ. Calif. Berkeley Publ. Bot. 68:1-71.

Nabata, S.I., Y. Nihara, M. Matsuya \& F. Takei. 1981. Ecological studies on Sargassum confusum from Rishiri Island in northern Hokkaido. Sci. Rep. Hokkaido Fish. Stat. 23:53-64.

Umezaki, I. 1983. Ecological studies of Sargassum miyabei Yendo in Maizuru Bay, Japan Sea. Bull. Jap. Soc. Sci. Fish. 49:1825-1834.

Umezaki, I. 1984. Ecological studies of Sargassum hemiphyllum C. Agardh in Obama Bay, Japan Sea. Bull. Jap. Soc. Sci. Fish. 50:1677-1683.

Yoshida, T. 1960. On the growth rings found in the root of Sargassum ringgoldianum Harvey (Fucales). Bull. Jap. Soc. Sci. Fish. 26:673-678. 